\title{
Energy based Self-Organizing Tree-based Routing Strategy for Wireless Sensor Networks
}

\author{
Navpreet Kaur \\ M.Tech. Scholar (CSE) \\ Shaheed Bhagat Singh State \\ Technical Campus, Ferozepur, \\ Punjab, India
}

\author{
Vishal Kumar Arora \\ Assistant Professor (CSE Dept.) \\ Shaheed Bhagat Singh State \\ Technical Campus, Ferozepur, \\ Punjab, India
}

\author{
Richa Sawhney \\ Lecturer \\ Shaheed Bhagat Singh State \\ Technical Campus, Ferozepur, \\ Punjab, India
}

\begin{abstract}
WSNs have gained a significant interest in space of analysis due to its big scope of applications. However energy consumption has become a significant limitation due to restricted battery power of sensor nodes. Although several protocols has been proposed so far to enhance the energy efficiency however still a lot of improvement can be done. This paper has proposed a new energy balanced, selforganizing, and cluster tree based routing protocol for wireless sensor networks. The projected technique utilize the leach based clustering protocol and improves the GSTEB additional by increasing the network lifetime. The experimental results have shown the substantial improvements.
\end{abstract}

\section{Keywords}

Energy-balance, network lifetime, clustering, self organized.

\section{INTRODUCTION}

Wireless Sensor Networks (WSNs) is taken into account as the foremost powerful technologies in twenty first century. Recent advancement in Micro-electronic-mechanical-systems (MEMS) and wireless communication system, tiny, low-cost and sensible nodes collaborated with wireless links and therefore the web deployed in physical space which offer a number of opportunities in several applications, as an example battle field police investigation, atmosphere observance, and health care applications.

A sensor network consists of a large number of sensor nodes that are tiny, light-weight and moveable. Each sensor node is provided with a transducer, microcomputer, transceiver and power supply. The transducer generates electrical signals by detecting physical effects and phenomena. The microcomputer processes and stores the device output. The transceiver receives commands from a central computer and transmits information there to central computer. The energy for every sensor node comes from electric battery supply that is confined.

WSNs are not centralized one as no static infrastructure exists. Peer-to-peer communication exists between nodes. Multihopping will cause the node to communicate with a node that is not in radio range of each other via intermediate nodes. Thus WSN provides flexibility of adding or removing nodes within the network. The network may be divided into no. of clusters. In every cluster, one amongst the sensor nodes is elected as Cluster Head $(\mathrm{CH})$ and therefore the remainder of the nodes act as Cluster Members (CM). All sensor nodes work in cooperation among every cluster to serve the request. Cluster head collects the information from its members and

information aggregation is completed by every cluster head to get rid of data redundancy and forwarded to the sink. As cluster head consumes additional energy than cluster members, the employment of cluster heads is distributed among all nodes in wireless network by rotating their roles to equalize energy consumption referred to as Cluster Head rotation.

Energy consumption is a vital issue in WSN as a result of sensor nodes being battery operated and cannot function while enough power is not available. The ever dynamical topology and restricted power-supply nodes create WSN quite difficult and become a preferred analysis space.

Characteristics of WSNs: Unlike traditional wireless sensor networks like MANETs, WSN has distinctive characteristics as follows:

Dynamic Network Topology: The network topology changes often as nodes are added or removed, node failure, energy depletion, or channel weakening.

Application Specific: The design of the network can be varied according to the required application.

Energy constrained: Nodes are moveable and are having confined energy, computation and storage capacities. This can be the important design consideration of WSN.

Self-configurable: Nodes are arbitrarily deployed without careful designing. Once deployed, nodes ought to configure autonomously themselves into a communication network.

Imperative problems in sensor networks are as follows:

- The most imperative concern in wireless device networks (WSNs) is that which method ought to be utilized to collect data in skillful manner among slightest of power expenditure.

- Low energy consumption.

- Accuracy of selective information.

Sensor nodes are powered through battery that is impracticable to revitalize once consumed. Since routing eat up a lot of power, efficient routing strategy in sensor networks is important. To increase the lifetime of WSNs, energy management in given recital a requirement has become the chief aspect within the system. Since it is in a position of 
achieving unchanged interaction between individual and central computer, it is believed that WSNs are the key of artificial brain.

\section{RELATED WORK}

As we know the working procedure of WSN is collecting related information of the given task and sending to the Base Station [1]. The nodes are deployed randomly in the target area and the base station will be located far away from the sensing field. After finishing their task, the nodes will die due to the more energy consumption. To solve such energy inefficiency many protocols was proposed, some of them are LEACH, PEGASIS, HEED etc.

In the LEACH protocol some nodes are chosen as the cluster head or control nodes on random basis and other remaining nodes will be connected to those as child nodes. The control nodes will act as the parent nodes. All the sensed information of the child nodes will be collected by the cluster heads and through the cluster heads, the information is sent to the base station. Here the energy consumption will be more misbalancing due to more load on the cluster head nodes.

The HEED Protocol i.e., Hybrid energy efficient distributed clustering algorithm, is the improvised version of the LEACH in selecting the Cluster head $\mathrm{CH}$. At each time HEED protocols selects the $\mathrm{CH}$ based on the residual energy usage at the nodes. When we compare with HEED and LEACH, HEED will effectively prolongs the network life time and the drawback is consumption of energy is more.

To overcome the drawback of the HEED, PEGASIS [2] Protocols was proposed to use the optimized power and this can be done by using the Greedy Algorithm to form a chain model of the sensor nodes. This PEGASIS protocol is more power efficient protocol than the other protocols like LEACH. When we compare it with the PEGASIS we can see the PEGASIS is $300 \%$ more efficient than the LEACH.

We have some other protocols like TBC (Tree Based Clustering) and PEDAP which uses the tree based routing process. The TBC is similar to LEACH [3] and is somewhat improvised. In TBC, each node records the information of its neighbors and builds topography through computing. The drawback is that some nodes consume more energy which are located away from the base station. The PEDAP is similar to the PEGASIS protocol and is of improvised version. [9] The drawback is that this protocol builds the topology which will cause the large amount of waste of energy. The BS in the network needs to build the topography to collect the information of the sensor nodes of parent and child nodes, so delay will be there.

General Self-Organized Tree-based Energy Balance routing protocol (GSTEB) was proposed to overcome the drawbacks of the existing protocols where network gathers data from a territory time to time where each node often senses the area of interest and throws the information back to Base station. The key goal of GSTEB is to achieve an extended network lifetime for dissimilar applications. The simulation results show that once the information collected by device nodes is very correlative, GSTEB will modify the foundation and recreate the routing tree with very little delay and tiny energy utilization. So a better balanced load is earned compared with the protocols like LEACH, HEED, PEGASIS, PEDAP, and TBC. As a result of GSTEB being a self-organized protocol, it solely consumes a small extent of energy in each round to vary the topography for leveling the energy consumption. Entirely, leaf nodes will transmit information within the same TDMA interval owing the short sending delay. GSTEB extend the period of time by $100 \%$ to $300 \%$ when compared with PEGASIS. In some cases, the period of the ultimate node within the network is further increased. To a small minor transformation is formed to build the performance of GSTEB similar with the aim of PEDAP.

So GSTEB is more or less the best result. Since the information composed by sensors cannot be amalgamated, GSTEB presents an extra simple way to balance the network consignment. In existence, it is not easy to distribute the load uniformly on all nodes in such a case. But GSTEB wants base station to calculate the topography which will increase energy waste as well as longer delay and are acceptable when compared among the energy consumption and the time delay for information transmission. Simulation results show that GSTEB extend the network lifetime over $100 \%$ compared with HEED. To overcome all the drawbacks we proposed a protocol called "Energy Balanced Self Organizing Tree Based routing Protocol" (EBSOTB).

\section{NETWORK AND RADIO MODEL}

\subsection{Energy Model}

We have assumed the same radio model which has been used in earlier works. For the radio hardware, the transmitter dissipates energy to run the transmitter radio electronics and power amplifier, and the receiver dissipates energy to run the receive radio electronics as shown in figure and is given by Eelec $=50 \mathrm{~nJ} / \mathrm{bit}$. Eamp $=100 \mathrm{~nJ} / \mathrm{bit} / \mathrm{m}^{2}$ is required for amplification of the transmit signal. For the scenarios described in this project work, both the free space $\left(\mathrm{d}^{2}\right.$ power loss) and the multi path ( $\mathrm{d}^{4}$ power loss) channel models were used depending on the distance between the transmitter and the receiver. If the distance is less than a threshold, the free space (fs) model is used; otherwise, the multi path (mp) model is used. In order to transmit a message of k-bit packet size over a distance $d$, the cost of transmission energy (ETX) and receiving energy (ERX) are computed as given in figure 1 showing the radio model where $\mathrm{n}$ is 2 for free space and 4 for multi path routing.

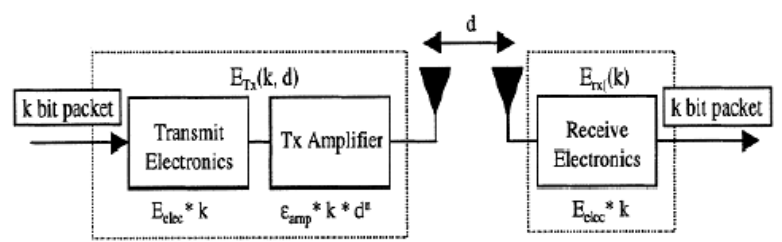

Fig 1: The radio energy dissipation model

There is an assumption in this model that the energy required to send data from node A to node B is same as energy required to send data from node $B$ to node $A$ for a given SNR ratio. To transmit a message over a short range distance such as between the sensor node and the cluster head, the energy dissipates by the transmit amplifier is directly proportional to d2. However for a long range distance such as between the cluster head and the base station, the energy dissipates by the transmit amplifier is directly proportional to $d 4$. The cost of transmission and reception for a message is high, so the protocol must tries to minimize the transmission distance and 
the number of transmit and receive operation required for sending a message.

\subsection{Network Design}

This wireless sensor network consists of immobile sensor nodes randomly deployed in a $100 * 100 \mathrm{~m}^{2}$ region. We consider the following characteristics of network:

- The network is designed by $\mathrm{N}$ sensor nodes randomly deployed in square field and has cluster hierarchical topology.

- Sensor nodes are homogeneous, immobile and energy constrained.

- The Base station is fixed and its location is predetermined which is far away from the network.

- In cluster, the cluster member nodes are cognizant of their cluster heads and can communicate directly with them.

- Energy consumed for sensing and processing the data is not considered.

- The cluster-head nodes communicate with their next-hop cluster-head, and finally far cluster-head nodes are communicated with BS.

\section{ENERGY-BALANCED SELF- ORGANIZING TREE-BASED ROUTING PROTOCOL}

In this research work, we proposed an EBSOTB (Energy Based Self Organizing Tree Based routing strategy) in which we mainly focused on how to enhance the network lifetime by minimizing energy consumption. To reduce and to balance the overall energy consumption, a multi-hop clustering technique is used for prolonging the network lifetime. For this, we selected cluster heads on the basis of parameters: maximum residual energy and less distance to BS. In EBSOTB protocol, along with the concept of clustering technique, the concepts of the existing GSTEB protocol are also used. This routing strategy minimizes the overall energy consumption by selecting the nearest neighbor to the node as its parent node and hence prolongs the network lifetime. The detailed research methodology of proposed EBSOTB routing protocol is defined in the figure 3.1 showing the flow chart of working of proposed protocol.

The proposed methodology is implemented in the following manner. The operation of this protocol is broken into rounds and each round consists of four phases: Initial Phase, Tree Constructing Phase, Self-Organized Data Collecting and Transmitting Phase, and Information Exchanging Phase.

\subsection{Initial Phase}

In Initial Phase, the network parameters are initialized. When Initial Phase begins, BS broadcasts a packet to all the nodes to inform them of beginning time, the length of time slot and the number of nodes N. Initial Phase is a significant preparation for the next phases. After Initial Phase, EBSOTB operates in rounds. In a round, the routing tree may need to be rebuilt and each sensor node generates a DATA_PAK that needs to be sent to BS. When BS receives the data of all sensor nodes, a round ended. Round is not a real time measurement unit, but it reflects the ability for transmitting the collected data for sensors, so round is a suitable time measurement unit for WSN lifetime. Each round contains three phases, including Tree Constructing Phase, Self-Organized Data Collecting and Transmitting Phase, and Information Exchanging Phase.

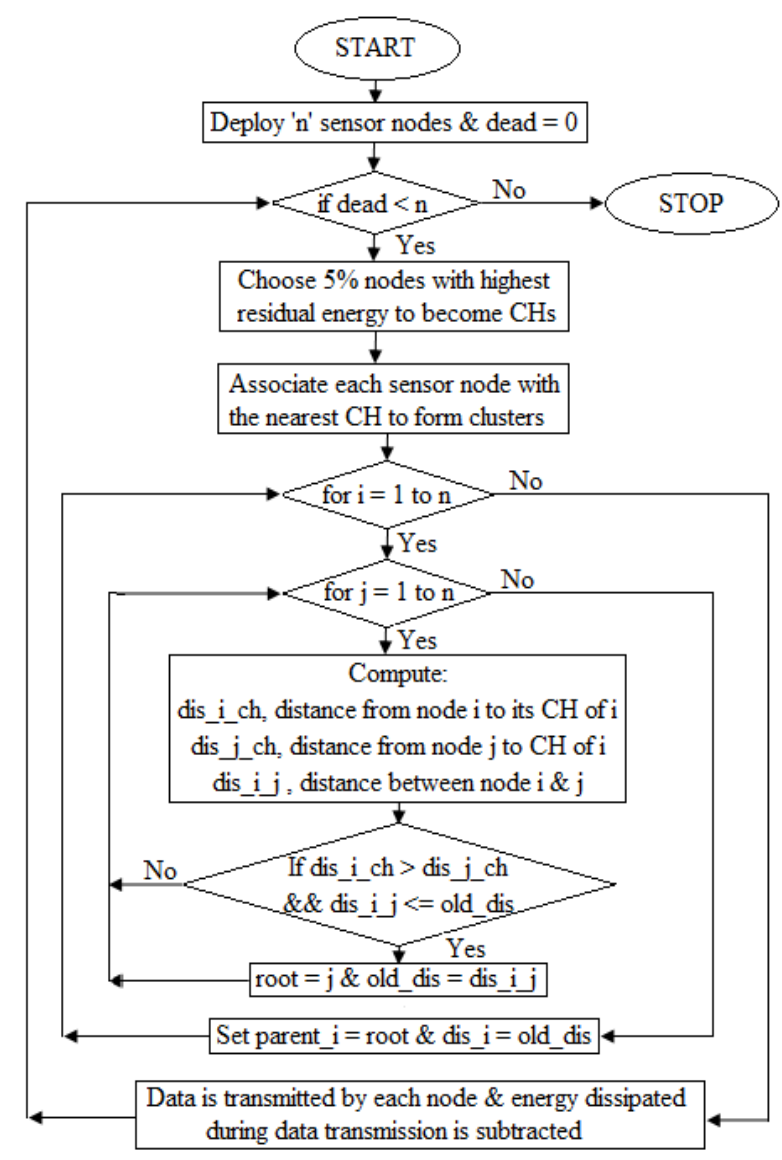

Fig 2: Flow-chart describing the working process of the proposed EBSOTB protocol.

\subsection{Tree Constructing Phase}

Within each round, EBSOTB performs the following steps to build a routing tree.

- BS assigns some nodes having the highest residual energy as $\mathrm{CHs}$ and broadcasts their ID and coordinates to all sensor nodes.

- $\quad$ Each node is assigned to nearest $\mathrm{CH}$ and thus forming clusters.

- Each cluster member node tries to select a nearest parent in its neighbors belonging to the same cluster using coordinates which are recorded earlier. The selection criteria are:

- For a sensor node, the distance between its parent node and the $\mathrm{CH}$ should be shorter than that between itself and the $\mathrm{CH}$.

- The process of Tree Constructing Phase can be regarded as an iterative algorithm. Choosing the parent node from all the neighboring nodes is based on energy consumptions. The relay node which 
causes minimum consumption will be chosen as the parent node. It is true that this relay node should choose its parent node in the same way. So a path with minimum energy consumption is found by iterations. If the sensor node cannot find a suitable parent node, it will transmit its data directly to its $\mathrm{CH}$.

- Because every node chooses the parent from its neighbors and every node records its neighbors' neighbors' information, each node can know all its neighbors' parent nodes by computing, and it can also know all its child nodes. If a node has no child node, it defines itself as a leaf node, from which the data transmitting begins. To build a routing tree we use BS to compute the topography. Even though we can fulfill this work without the control of BS, a large amount of energy is wasted in the next phase.

\subsection{Self-Organized Data Collecting and Transmitting Phase}

After the routing tree is constructed, each sensor node collects information to generate a DATA_PKT which needs to be transmitted to BS. Frequency Division Multiple Access (FDMA) or Code Division Multiple Access (CDMA), the schedule generated under competition is able to avoid collisions. However, the accompanying massive control packets will cause a large amount of energy to be wasted. By using the control of BS, the energy waste can be reduced and thus the process may be much simpler. At the beginning of each round, the operation is also divided into several time slots. In the time slot, the node whose ID is i turns on its radio and receives the message from BS. BS uses the same approach to construct the routing tree in each round, and then BS tells sensor nodes when to send or receive the data. In each TDMA time slot, the nodes work in turns defined by BS. When BS receives all the data, the network will start the next phase.

\subsection{Information Exchanging Phase}

BS can collect the initial energy and coordinates information of all the sensor nodes in Initial Phase. For each round, BS builds the routing tree and the schedule of the network by using the residual energy and coordinates information. Once the routing tree is built, the energy consumption of each sensor node in this round can be calculated by BS, thus the information needed for calculating the topology for the next round can be known in advance. However, because WSN may be deployed in an unfriendly environment, the actual residual energy of each sensor node may be different from the residual energy calculated by BS. To cope with this problem, each sensor node detects its actual residual energy in each round. When the two values of remaining energy of a sensor node are different, the sensor node generates an error flag and packs the information of actual residual energy into DATA_PKT, which needs to be sent to BS. When this DATA_PKT is received, BS will get the actual residual energy of this sensor node and use it to calculate the topology in the next round.

\section{COMPARATIVE ANALYSIS AND SIMULATION RESULTS}

A MATLAB simulation of EBSOTB is completed to estimate the performance. During this section we tend to estimate the performance of the projected EBSOTB strategy and compare it with the GSTEB on the idea of initial node dead time and all nodes dead time by varying the count of sensing nodes being deployed within the field from 100 to 300 . The research work tend to assume that the sensing nodes are distributed arbitrarily in a $100 * 100$ region and having 0.25 Joules of initial energy. The table- 1 defines all the parameters used throughout simulation. DATA_PKT length of 2000 bits is used and CTRL_PKT length of 100 bits is used.

Table 1. Simulation Parameters

\begin{tabular}{|c|c|}
\hline Parameters & Values \\
\hline Size of target area & $100 * 100 \mathrm{~m}^{2}$ \\
\hline Location of Base Station & At $(50,175)$ \\
\hline Number of sensor nodes deployed & $100-300$ \\
\hline Initial energy & $0.25 \mathrm{Joules}$ \\
\hline Transmitting / Receiving energy, $\varepsilon_{\text {elec }}$ & $50 \mathrm{~nJ} / \mathrm{bit}$ \\
\hline Data-aggregation energy, $\varepsilon_{d a}$ & $5 \mathrm{~nJ} / \mathrm{bit}$ \\
\hline Free-space energy, $\varepsilon_{f s}$ & $10 \mathrm{pJ} / \mathrm{bit} / \mathrm{m}^{2}$ \\
\hline Multipath energy, $\varepsilon_{m p}$ & $0.013 \mathrm{pJ} / \mathrm{bit} / \mathrm{m}^{4}$ \\
\hline Data packet size & $2000 \mathrm{bits}$ \\
\hline CH percentage & $5 \%$ \\
\hline
\end{tabular}

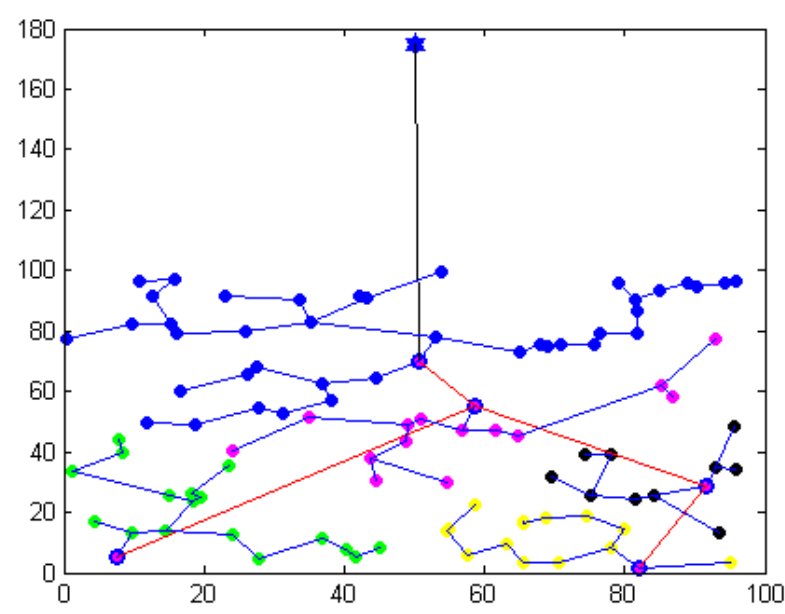

Fig 3: Routing Tree generated by EBSOTB protocol for 100 nodes randomly deployed in a square area.

The Figure 3 show the topography generated for communication of sensor nodes with the sink. Here clusters are shown with different colors of member nodes. The blue lines represents the intra-cluster communication in which each cluster member nodes communicate with its nearest neighbor belonging to the same cluster to transmit its sensed data to the corresponding $\mathrm{CH}$. The red lines represent the inter-cluster communication in which the $\mathrm{CHs}$ communicate with other nearest $\mathrm{CH}$ which is closer to the BS. And finally, the black line represents the communication of main $\mathrm{CH}$ with the $\mathrm{BS}$ directly which is located far away from the sensing field.

After few seconds, few of the sensing nodes drain out all their energy and become dead. The reason why some of the nodes 
drained out their energy before the others is because these nodes would have become $\mathrm{CHs}$ in the initial rounds of data transmission. After a number of simulations, we implemented our algorithm on the basis of above network parameters. The following results were gathered.

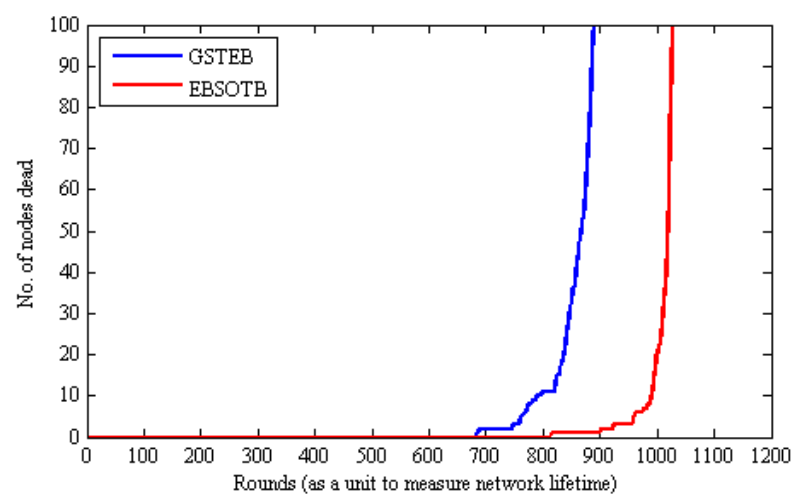

Fig 4: Graph showing the comparison of number of nodes being dead for the GSTEB and proposed EBSOTB protocol.

Figure 4 shows the number of dead nodes of GSTEB and the proposed EBSOTB strategy. EBSOTB clearly continue for many rounds more than the other clustered routing protocols because this proposed protocol have better policy for $\mathrm{CH}$ selection.

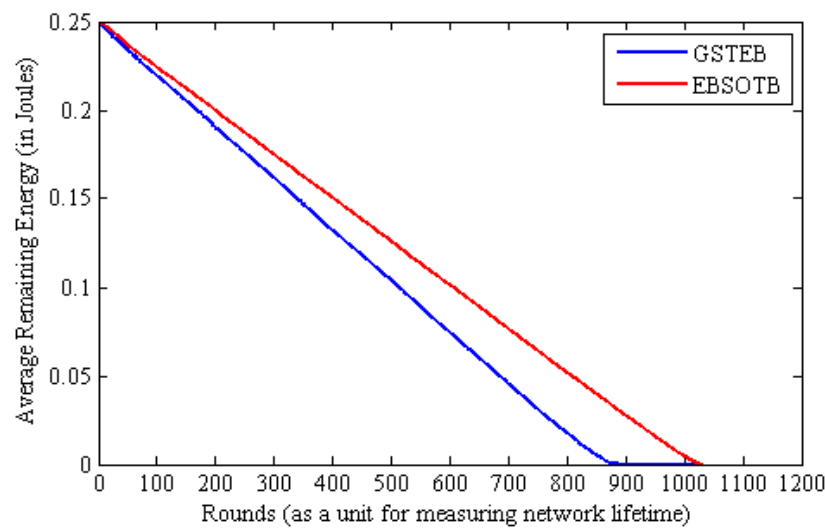

Fig 5: Graph representing comparison of the average remaining energy of sensor network for GSTEB and proposed EBSOTB protocol.

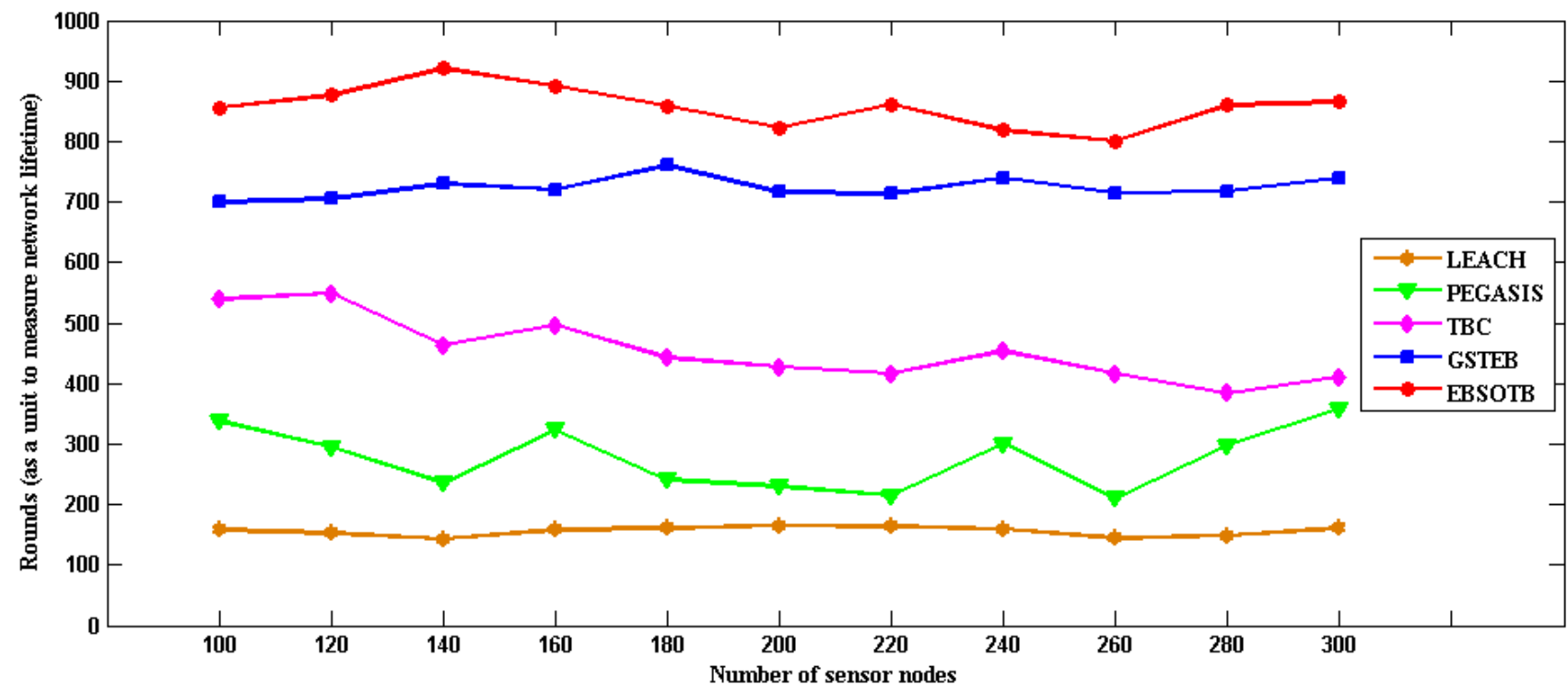

Fig 6: Graph showing the comparison when first node dies in LEACH, PEGASIS, TBC, GSTEB and EBSOTB for the number of nodes from 100 to 300 in the square area where round is considered as the time measurement unit.

In Figure 5, the comparison between the remaining energy of nodes after transmission of data to BS with number of rounds is shown. The remaining energy of the sensor node is the residual energy which remains after performing the tasks for that particular round. In the figure 5, we have observed that the proposed EBSOTB protocol has less energy dissipation than the GSTEB.

Figure 6 shows the network lifetime for different number of nodes from 100 to 300 by keeping the initial energy of each sensor node same i.e. 0.25 Joule for different protocols. The results clearly show that the proposed EBSOTB protocol work good for denser network also.

\section{CONCLUSION AND FUTURE SCOPE}

The simulation results clearly illustrates that by implementing clustering approach in the GSTEB protocol gives much better results than earlier. Hence the proposed Self-Organized Energy-Balance Clustering protocol is better than the existing GSTEB protocol. The lifetime of the wireless network can further be increased by minimizing the communication distances among the sensor nodes for data transmission.

\section{REFERENCES}

[1] Zhao Han, Jie Wu, Member, IEEE, Jie Zhang, Liefeng Liu, and Kaiyun Tian, "A General Self-Organized Tree- 
Based Energy-Balance Routing Protocol for Wireless Sensor Network", IEEE 2014

[2] Vishal Kumar Arora (Research Scholar), Vishal Sharma, Monika Sachdeva, "A survey on LEACH and other's routing protocols in wireless sensor network", Optik 127 (2016) 6590-6600

[3] Manjinder Kaur and Dr. Shashi B. Rana, "Performance Comparison of Leach and GSTEB in Wireless Sensor Network ",IJERT 2015

[4] Wendi Rabiner Heinzelman, Anantha Chandrakasan, and Hari Balakrishnan, "Energy-Efficient Communication Protocol for Wireless Micro sensor Networks". IEEE 2012

[5] Shikha Chhabra,Vishal Arora,"A Review on General Self-Organized Tree-Based Energy-Balance Routing Protocol For Wireless Sensor Network"ISSN 2277-3061, Volume 16 Number 2, International journal of computers \& Technology.

[6] S. Lindsey and C. S. Raghavendra, "PEGASIS: Powerefficient gathering in sensor information systems," in IEEE Aerospace Conference Proceedings, vol. 3, pp. 1125-1130, March 2002.

[7] Gurpreet Kaur, Vishal Arora," A Modified LEACH Algorithm using Hard and Soft Threshold in Wireless Sensor Network: A Review" ISSN 2277-3061.

[8] Gurpreet Kaur, Vishal Arora, "Enhancement of network lifetime using improved MOD-LEACH in WSN", International Journals of Computers \& Distributed Systems, ISSN 2278-5183, Volume 1 Number 4

[9] SHA Chao, WANG Ru-chuan, HUANG Hai-ping, SUN Li-juan1," Energy efficient clustering algorithm for data aggregation in wireless sensor networks", ScienceDirect 2010

[10] Elkamel Rabiaa, Baccar Noura, Cherif Adnene, "Improvements in LEACH based on K-means and Gauss algorithms". Elsevier 2015

[11] Palak Agrawal, P.R.Pardhi, "Routing Protocols For WSN (General Self Organized Tree Based Routing Protocols)", International Journal Of Computer Science And Applications, Vol. 8, No.1, Jan-Mar 2015

[12] Jin Zhou, Yuan Zhang, Yuyan Jiang, C. L. Philip Chen, Long Chen, "A Distributed K-means Clustering Algorithm in Wireless Sensor Networks" ICCSS 2015

[13] N. Sepideh, N. Aras, A. Fairouz "SLTP: Scalable Lightweight Time Synchronization Protocol for Wireless Sensor Network", Springer - Verlag Berlin Heidelberg 2007.

[14] W. Heinzelman, A. Chandrakasan and H. Balakrishnan, An applicationspecific protocol architecture for wireless micro sensor networks, IEEE Transaction on Wireless Communications, vol. 1, pp. 660-670, 2002.

[15] M. Aslam, M. B. Rasheed, T. Shah, A. Rahim, Z. A. Khan, U. Qasim, M. W. Qasim, A. Hassan, A. Khan, N. Javaid, "Energy optimization and Performance Analysis of Cluster Based Routing Protocols Extended from LEACH for WSNs",

[16] Meenakshi Sharma, Anil Kumar Shaw, "Transmission Time and Throughput analysis of EEE LEACH, LEACH and Direct Transmission Protocol: A Simulation Based Approach", Advanced Computing: An International Journal (ACIJ), Vol.3, No.5, September 2012.

[17] F. Xiangning and S. Yulin, Improvement on LEACH protocol of wireless sensor network, 2007 IEEE International Conference on Sensor Technologies and Applicat ions

[18] Muhammad Omer Farooq, Abdul Basit Dogar and Ghalib Asadullah Shah, MR-LEACH: Multi-hop Routing with Low Energy Adaptive Clustering Hierarchy, 2010 IEEE Computer Society, SENSORCOMM.

[19] W. Liu and J.Yu, Energy efficient clustering and routing scheme for wireless sensor networks, IEEE 2009.

[20] Mayank Chandra, Prashant Saini, Manju Khari, C. S. Vinitha, Clustering In Wireless Sensor Networks: Performance Comparison Of Leach And Multi-Hop Leach Protocols, Proceedings of 24th IRF International Conference, 3rd May 2015, Chennai, India.

[21] Ashlyn Antoo, Rameez Mohammed A, EEM-LEACH: Energy Efficient Multi-hop LEACH Routing Protocol for Clustered WSNs, 2014 IEEE, International Conference on Control, Instrumentation, Communication and Computational Technologies (ICCICCT).

[22] Imen Souid, Haithem Ben Chikha, Malika El Monser, Sondes Gasmi and Rabah Attia, "Multi-Hop Dynamic Clustering LEACH Protocol for Large Scale Networks", 2014 IEEE.

[23] "An Energy-balanced Distributed Clustering Protocol for Wireless Sensor Networks" by Saman Siavoshi, Yousef S. Kavian, Mehdi Tarhani. 2014 IEEE.

[24] "A Survey on Routing Protocols in Wireless Sensor Networks" by M. Patil and Rajashekhar C. Biradar. $18^{\text {th }}$ IEEE International Conference on Networks (ICON), 2012.

[25] "MS-Leach: A Routing Protocol Combining Multi-hop Transmissions and Single-hop Transmissions" by Tang qiang, Wang bingwen, Dai zhicheng. IEEE 2009 PacificAsia Conference on Circuits, Communications and System.

[26] "A survey on routing protocols for wireless sensor networks" by Kemal Akkaya \& Mohamed Younis. 2003 Elsevier.

[27] "A systematic review on clustering and routing techniques based upon LEACH protocol for wireless sensor networks" by Sudhanshu Tyagi , Neeraj Kumar. 2012 Elsevier.

[28] "Survey of Extended LEACH-Based Clustering Routing Protocols for Wireless Sensor Networks" by M. Aslam, N. Javaid, A. Rahim, U. Nazir, A. Bibi, Z. A. Khan. 14th International Conference on High Performance Computing and Communications, 2012 IEEE 